BMJ Quality

\section{Absconding: reducing failure to return in adult mental health wards}

To cite: Bailey J, Page B, Ndimande $\mathrm{N}$, et al. Absconding: reducing failure to return in adult mental health wards. BMJ Quality Improvement Reports 2016;5:u209837.w5117. doi:10.1136/bmjquality. u209837.w5117

Received 1 June 2016 Revised 16 September 2016 Published Online First 31 October 2016

\section{ABSTRACT}

Failing to return from leave from acute psychiatric wards can have a range of negative consequences for patients, relatives and staff. This study used quality improvement methodology to improve the processes around patient leave and time away from the ward. The aim of this study was to improve rates of on-time return from leave by detained and informal patients by $50 \%$.

Following a baseline period, four interventions were implemented and refined using PDSA cycles. The main outcome measure was the proportion of periods of leave where the patient returned on time. Late return was defined as failure to return to the ward within 10 minutes of the agreed time.

At baseline, the rate for on-time return was $56.0 \%$; this increased to $87.1 \%$ post-intervention, a statistically significant increase of $55.5 \%$. SPC charts show that the interventions were associated with improvements. The improvements have been sustained and the interventions are fully embedded into daily practice. The project was refined to local context and trialled on six additional wards: four of the six wards have successfully implemented the interventions and have on-time return rates of over $90 \%$.

This project produced a marked and sustained improvement in patients returning on-time from leave, facilitating a more open discussion between staff and patients about the purpose and value of periods away from the ward. Quality improvement approaches can be effectively applied in mental health settings.

\section{PROBLEM}

Absconding, or absence from a mental health facility without permission, is a significant safety issue that can have a range of negative consequences for patients, their relatives, and staff. Absconding includes both leaving the ward without permission and failure to return at an agreed time. In some instances, the consequences of absconding can be catastrophic: between 2003 and 2013, $22 \%$ of inpatient suicides in England occurred following an incident of absconding from mental health wards. ${ }^{1}$ Patients who are absent without permission are also at greater risk of self-harm, self-neglect, missed medication, and interruptions to treatment plans. $^{2}$ Absconding incidents can also cause relatives and staff much distress and anxiety, ${ }^{3}{ }^{4}$ can lead to a deterioration in the relationship between staff and patients' relatives, and distract nurses from other responsibilities. In many cases the police are also engaged in the search for the missing patient which has a considerable impact on police resources. $^{56}$

The setting for this improvement project is a UK National Health Service (NHS) Trust which provides community and mental health services. The project was initially driven by two serious incidents in the Trust during time away from the ward. Analysis of the official database of absent-without-leave (AWOL) incidents revealed that the majority of absconding incidents on adult acute wards in the Trust were cases of patients failing to return from leave rather than leaving without permission. Our primary aim was therefore to produce a $50 \%$ increase in the numbers of all patients, both voluntary and detained, returning at the time agreed.

The project was initially developed and tested in a single lead site (ward A). We provide a detailed description of the implementation process and results for this ward. The interventions developed were then further disseminated and tested on six additional wards within the organisation. All wards involved in this project are 20 bedded adult psychiatric wards; the majority are single sex acute wards and one is a mixed sex rehabilitation ward. The wards vary in design (a Victorian building, a fifteen year old building and a purpose-built site). All wards have safe staffing levels but some staff turnover issues. The majority of patients are detained under the Mental Health Act (formal patients) and typically up to a quarter are admitted on a voluntary basis (informal patients). In either case, most patients are allowed leave as soon as they begin to recover so that they can return home and re-establish their ordinary lives as far as possible; in some cases the leave is required so that they can look for suitable 
accommodation and re-adapt to living at home. In the UK, leave is an important part of the legal framework and ethos. ${ }^{7}$ Nurses and health care assistants on the wards typically manage 20-25 periods of leave a day; following the recently introduced no smoking policy, this has increased further.

\section{BACKGROUND}

Based on the literature, the extent of absconding is hard to quantify. There is no universally agreed definition for absconding nor an agreed method for calculating rates. $^{3} 8$ The majority of prevalence studies do not define absconding, ${ }^{8}$ and those that do use varying definitions of absence without permission that range from 1 hour to "midnight on the day in question". ${ }^{9}{ }^{10}$ Studies also differ in whether they included or excluded voluntary patients. ${ }^{2} 8$ Critically the majority of prevalence studies rely on retrospective analysis of incident reporting systems, which are notoriously unreliable measures of true rates. ${ }^{10-12}$ Although the true extent of absconding remains unknown, these are clearly high-risk events and sufficiently frequent to cause concern.

In spite of the serious risk to patients who fail to return from leave, there have been very few attempts to systematically reduce absconding. ${ }^{3}$ In the UK, a wideranging "anti-absconding package" was trialled in twenty psychiatric wards and led to an overall 25\% decrease in absconding. ${ }^{13}{ }^{14}$ Interventions included: a signing in and out book, supportive breaking of bad news, postincident debriefing of patients, multidisciplinary review, identification of patients at high risk of absconding, targeted nursing time daily for high risk patients, and facilitated social contact for those at risk of absconding. ${ }^{13} 14$ The success of the package was mixed: some wards made significant improvements but others saw increases in their absconding rate. ${ }^{13}{ }^{14}$ The follow-up period was short so we do not know whether the observed improvements were sustained. We aimed to develop a focussed intervention which built on previous understanding but placed a stronger emphasis on: i) staff and patient engagement in the development of the intervention and ii) fostering a responsive relationship between staff and patients rather than treating absconding simply as a security issue.

\section{BASELINE MEASUREMENT}

The outcome measure was the proportion of occasions where the patient returned on time in a given week. Late return was defined as "any inpatient, detained or informal, who fails to return to the ward later than 10 minutes over the leave period that was agreed and documented by ward staff, and has not made contact with the ward to agree a later return time". A signing in and out book was the data source.

Baseline data ( 17 weeks) for ward A indicated that the ward managed a mean of 165 periods of leave per week. The mean proportion of patients returning on time was
$56.0 \%$. There was considerable variability in the baseline data, with weekly rates of on-time return ranging from $30.2 \%$ to $75.5 \%$. This data suggests that over 1000 periods of leave per week are managed across nonforensic adult mental health wards in the organisation.

\section{DESIGN}

Before the start of the quality improvement process, a clear procedure for late return was specified and agreed across the organisation. If a patient did not return at the agreed time, a 10 minute search period was to be initiated during which ward staff would search the hospital grounds, attempt to contact the patient and the patient's family and friends and if the patient remained uncontactable, ring the police.

A cross-section of staff (ward matrons, ward managers, nurses, health care assistants) from three wards across the organisation agreed upon four interventions. The team was led by the quality improvement lead for the organisation. The agreed interventions were: i) a signing in and out book for patients going on leave, ii) ward phone cards handed to patients before they leave including time due back, iii) patient information leaflets about leave for both formal and informal patients and iv), pre-leave forms to be completed by patients in advance recording the purpose of leave, support arrangements and agreed time of return. Current inpatients on ward A were engaged in the planning and development of the project: their ideas and suggested adaptions to the interventions were gathered in daily community meetings. Ward staff were trained in Institute for Healthcare Improvement (IHI) methodology and measurement for improvement.

The primary data source was the signing in and out book; data was collected and reviewed weekly. Plan-do-study-act (PDSA) cycles were used to implement and refine the interventions. The data was shared in weekly staff meetings and any changes to the interventions were discussed collectively. Training of staff was key to ensuring the accuracy and completeness of data. Throughout the measurement period there were numerous changes in staff and patients. However staff remained motivated and engaged and the project was sustained by the leadership and experience of the senior nurse.

Statistical process control (SPC) charts were used to determine the effectiveness of the tests of change: the proportion of periods of leave where the patient returned on time was plotted by week throughout the study period. Both an xmr-chart and a p-chart were plotted and the decision made as to which type of SPC chart was most suitable for the data. ${ }^{15}$ The opportunity arose post project to assess the statistical significance of the improvement: the data was analysed pre and post intervention using chi-square analysis. A $p$ value of $<0.05$ was considered significant. 


\section{STRATEGY}

Four interventions in the leave process were introduced and refined using frequent PDSA cycles, often with small changes each time based upon staff and patient feedback. We describe in the detail the four interventions and a few example PDSA cycles used to refine them. The resources for each intervention are attached as an appendix.

Signing in and out book

The aim of the first intervention was to develop a fast and accurate method for recording patient leave. On ward A the signing in and out book took the form of an A3 register in which each instance of leave was recorded, detailing the patient's name, agreed time of leave and return, where the patient was going, what they were wearing and any items they took with them. PDSA cycles were used to trial adjustments to the layout of the A3 sheets to ensure it was quick and easy to complete accurately and captures all information that is helpful to support the leave process. In another PDSA cycle posters were placed on the wall at ward entrance and in the ward office to remind staff to sign patients in and out. Over time the use of the A3 register became routine for staff and patients with staff responding proactively and checking every 15 minutes on which patients were due to return.

Ward phone card

The aim of the ward phone cards was to prompt patients to return on time. All patients were offered ward phone cards when they went on leave. The cards detailed the address of the ward, the Trust logo, the ward phone number and the time the patient was due back. Initially all patients received a card every time they went on leave. On the basis of patient and staff feedback a PDSA cycle was trialled where cards were always available but patients were not required to take a new one each time they went out on leave: some patients did not need the cards and others were confused between the time of return on old and new cards.

Patient information leaflets

The aim of the patient information leaflets was to explain the arrangements and expectations surrounding leave and time away from the ward, whilst also giving a message of care. Separate leaflets were created for detained and informal patients. Although informal patients are free to leave at any time, they were asked to remain on the ward at night between the hours of $9 \mathrm{pm}$ and $9 \mathrm{am}$, at medication times, for agreed meetings, ward reviews, planned therapeutic activities, and unless otherwise agreed, at meal times. Both escorted and unescorted leave for detained patients must be agreed by the consultant psychiatrist. The leaflets were regularly discussed with patients to remind them of the procedures surrounding leave.

Pre-leave form

The aim of the pre-leave form was to discuss, together with the patient, the therapeutic aim and duration of the leave or time away from the ward. The discussion provided an ideal opportunity to clarify aspects that had, on occasion, previously been poorly addressed such as transport arrangements, time needed to complete tasks, the relationship with those supporting the patient whilst on leave, the availability of necessary monies, and the importance of contacting the ward in the event of any change to the agreed return time. The agreement reached between patient and staff was recorded on the pre-leave form. PDSA cycles were used to adapt the format of the pre-leave form (See supplementary Resources: signing in and out sheet, ward phone card, patient information leaflets and pre-leave form).

\section{RESULTS}

The specific aim of the study was to increase rates of on-time return from leave or time away by $50 \%$. During baseline (17 weeks), the mean rate of on-time return was $56.0 \%$. This increased to $87.1 \%$ post-intervention (45 weeks). This improvement corresponds to a $55.5 \%$ increase.

Figure 1 shows an xmr-chart (SPC chart) for the 18 month study period (12th May 2014 - 2nd November 2015), including baseline, intervention and postintervention (consisting of an embedding and a sustaining period). During the baseline period (17 weeks), there was considerable variability in the data, as reflected in the wide control limits, with weekly rates of on-time return ranging from $30.2 \%$ to $75.5 \%$. The interventions were implemented one at a time over a 16 week period: the mean rate of on-time return during this intervention period was $73.3 \%$.

From January 2015 no new interventions were implemented and the focus of activity in the next six months turned to refining the interventions using PDSA cycles and embedding the changes into daily practice. Changes were sustained and variability in rates of return on time reduced, as can be seen in the narrowing of the control limits in this period. The mean proportion of on-time returns during the first six months of 2015 was $83.0 \%$ which increased to $91.0 \%$ in the remainder of the year. We have described the first period as one of embedding the changes and the second as one of sustaining the gains (Figure 1). The narrowing of the upper and lower control limits between the embedding and sustaining period indicates an increase in reliability. The xmr-chart demonstrates that the improvement was sustained for a further four months.

To determine the statistical significance of the improvement, the baseline period was compared against the full post-intervention period (embedding and sustaining) using chi-squared analysis. The unit of analysis was periods of leave rather than patients. The proportion of periods of leave where the patient returned on time was significantly higher post intervention compared to baseline, $\mathrm{x}^{\wedge 2}(1)=1412.7, \mathrm{p}<0.001$.

The number of periods of leave authorised or agreed with the patient per week increased dramatically in April 


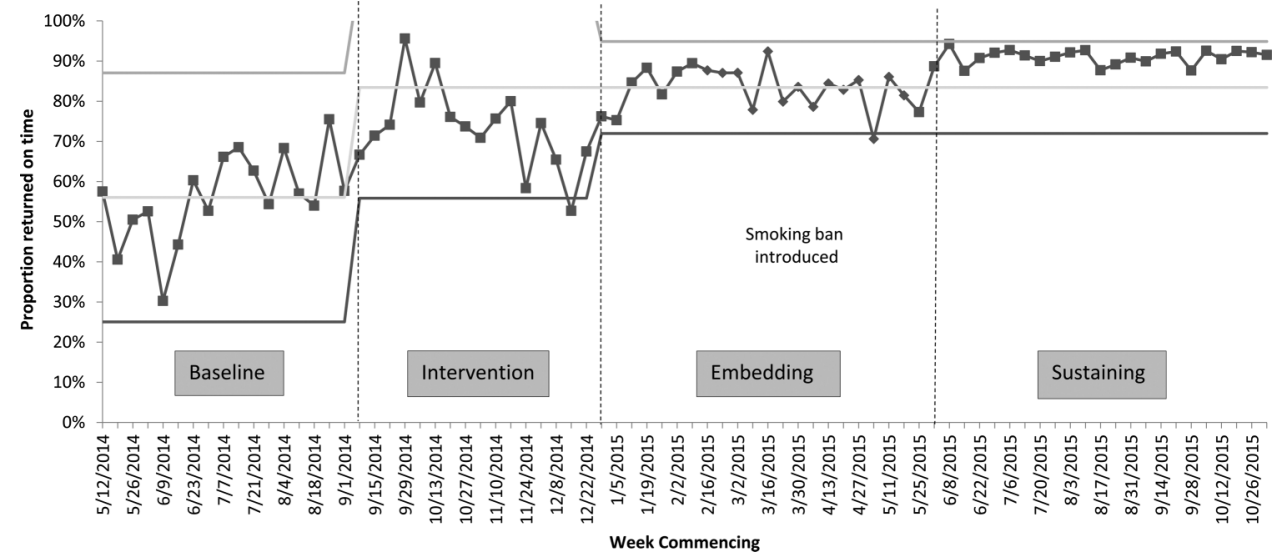

Figure 1 xmr-chart showing the weekly proportion of periods of leave where the patient returned on time. The chart is split into four periods: baseline, intervention, embedding and sustaining.

2015. This was almost certainly the result of the introduction of a non-smoking policy on April 1st 2015: from this point forward, if patients wanted to smoke they required authorised or agreed leave in order to leave the site as they could no longer smoke within the hospital grounds. The immediate result was an increase in requests for short periods of absence. Before the introduction of the non-smoking policy, a mean of 176 periods of leave were taken each week on the ward. This increased to a mean of 346 periods of leave post the introduction of the non-smoking policy, an increase of $97 \%$. Although the non-smoking policy greatly increased the number of short periods of leave, there appeared to be little impact on the overall rate of patients returning on time (see Figure 1).

Spread to other wards

The interventions were sequentially adopted on six other acute mental health wards, in the same organisation of varying patient population and building design. A new aim was set of achieving $95 \%$ on time return for all wards. Table 1 presents the baseline and most recent six months of data (mid-September 2015 - mid-March

Table 1 Comparison of baseline on time return rate with on time return for the most recent six months of data on the six additional wards (mid Sept 2015- mid March 2016).

\begin{tabular}{lll}
\hline Type of ward & $\begin{array}{l}\text { Baseline }^{\mathrm{a}} \\
(\%)\end{array}$ & $\begin{array}{l}\text { On time return rate } \\
(\%)\end{array}$ \\
\hline Male acute & 30.0 & 92.4 \\
Rehabilitation & $71.1^{\mathrm{a}}$ & 95.4 \\
mixed & & \\
Male acute & $64.6^{\mathrm{a}}$ & 75.5 \\
Female acute & $77.4^{\mathrm{a}}$ & 90.7 \\
Female acute & 27.8 & $68.2^{\mathrm{b}}$ \\
Male acute & 34.0 & 90.2 \\
\hline
\end{tabular}

Where the exact dates of the baseline period are not known, the first 12 weeks of data collection are taken as the baseline.

${ }^{\mathrm{b}}$ The intervention had to be re-started on this ward. The most recent 12 weeks of data is presented.
2016) for these wards. Baseline data varied in quality, ranging from $27.8 \%$ to $77.4 \%$ on-time return, with a mean of $50.8 \%$. To-date four of the six wards have fully implemented the interventions. These four wards all have on-time return rates of above $90 \%$ for the most recent six months. Attempts to re-introduce the intervention and improve rates of on-time return continue in the remaining two wards, with one achieving an on-time return rate of $75.5 \%$. Improvement in the remaining ward was interrupted at a critical stage when the improvement leader left the organisation and consequently the interventions have had to be restarted on this ward.

\section{LESSONS AND LIMITATIONS}

Our study utilised quality improvement methodology, allowing staff some flexibility in adapting the interventions to their own context and giving them ownership of the project. Staff were trained in IHI methodology, received fortnightly coaching from trained Patient Safety Leads, collected their own real-time data and reviewed their own progress. The project benefitted from strong board engagement, safety team leadership, and local nursing leadership. The lead site in particular profited from the strong leadership and determination of the matron and her commitment to supporting staff in developing skills in improvement methodology. Unlike previous studies on absconding, our study used a clear definition of late return and clear data collection procedures, rather than relying on retrospective analysis of official reporting systems which have frequently been cited as unreliable and imprecise data sources. ${ }^{8} 10$

The coaching approach in which a Patient Safety Lead trained in IHI methodology visited the ward every two weeks for the first year was resource intensive; it is likely that progress would be slower and more uncertain if less support was available. This project was the first to use quality improvement methodology in these settings and there was little initial understanding or experience of 
these methodologies. Subsequent projects with a core of knowledgeable staff could produce more quickly. The combination of interventions has created substantial and sustained improvement. However it is difficult to assess the relative effect of each as they were introduced over a short time period. Nonetheless, the data suggests that this relatively simple bundle of interventions can substantially reduce instances of failure to return to the ward.

This quality improvement project is applicable to psychiatric wards which manage regular patient leave; although we have only tested this project on adult acute wards and a rehabilitation ward, we believe it may be suitable for other types of wards which manage leave, such as forensic wards, adolescent units or learning disability settings. However, from our experience, strong ward leadership, regular coaching, patient collaboration and board level support are needed to ensure successful implementation. This project was successful despite staff turnover issues; however, implementation would inevitably be faster with consistent staffing. Training of staff and strong leadership were key. Collecting baseline data on how often patients return late from leave can provide important learning about the safety and reliability of local leave processes, and the impetus to commence quality improvement work like this.

Interestingly, the introduction of the non-smoking policy did not lead to a big change in reliability of the process, nor can it explain the success of the intervention, since improvement pre-dated the introduction of the no smoking policy. The ability of the system to cope with such an increase in the periods of leave authorised per week demonstrates that the process was sufficiently embedded to tolerate significant disturbances/changes. The data collection method (the signing in and out book) was adapted and improved as the project progressed because it was an intervention in itself. Improvements in data completion may, in part, explain the improvements in patients returning on time.

\section{CONCLUSION}

The interventions trialled were associated with highly significant improvements in the proportion of patients returning on time from leave or time away from the ward. On the lead ward only $56.0 \%$ of patients returned on time during baseline improving to $91.0 \%$ in the final sustaining period over one year later. The project was subsequently spread to a further six wards, four of which fully implemented the tests of change and have sustained on time return rates of above $90 \%$.

The reduction in the number of patients returning late from leave has reduced staff time on searching for missing patients considerably and has saved considerable but unquantified police time. The project has also prompted a policy change (the incorporation of a 10 minute search period) and there are now clear procedures for absconding across the organisation with increased adherence to local policy. Where patients have failed to return from leave or time away from the ward, local inquiry has shown that staff have followed the new procedures, including the required reporting, according to the policy.

Furthermore, staff report more discussion about leave with patients and amongst staff, and that patients feel more empowered to discuss their leave. Staff also report that patients' attitudes to leave have changed, from seeing it as restrictive, to being an important part of their care, which they are involved in negotiating and agreeing. Conversations about leave have moved from primarily concerning rules and sanctions to open discussions about the purpose and value of being away from the ward, inquiries about support while away and how to access help and support when difficulties arise.

Currently all wards continue to collect and review their data weekly. The Safer Care Team receive the data and provide support with data analysis and question any unexpected patterns in the data. The coaching frequency has been reduced. The next step will be to trial reducing the frequency of data collection to see if the tests of change remain part of daily practice. The project has recently been further adopted in wards in a neighbouring Trust.

Patient safety in mental health is still an emerging field. The available literature consists mostly of opinion pieces and small descriptive and largely retrospective studies. ${ }^{16}$ Safety needs to be approached differently in mental health from the acute hospital sector, but it is encouraging that quality improvement methods can be successfully applied in this context and embraced as being of value by both staff and patients. This project produced a marked and sustained improvement in patients returning on time from leave facilitating a more open discussion between staff and patients about the purpose and value of periods away from the ward.

Acknowledgements We thank all the ward teams and patients who participated in this quality improvement work.

Declaration of interests Nothing to declare.

Ethical approval Ethical approval for the project was agreed through the Safer Care Programme Steering Board, chaired by the Director of Nursing and Clinical Standards (DNCL).

Open Access This is an open-access article distributed under the terms of the Creative Commons Attribution Non-commercial License, which permits use, distribution, and reproduction in any medium, provided the original work is properly cited, the use is non commercial and is otherwise in compliance with the license. See:

- http://creativecommons.org/licenses/by-nc/2.0/

- http://creativecommons.org/licenses/by-nc/2.0/legalcode

\section{REFERENCES}

1. The National Confidential Inquiry into Suicide and Homicide by People with Mental IIIness Annual Report 2015: England, Northern Ireland, Scotland and Wales July 2015. http://www.bbmh. manchester.ac.uk/cmhs/research/centreforsuicideprevention/nci/ reports/NCISHReport2015bookmarked2.pdf (accessed 18 Jan 2016).

2. Bowers L, Jarrett M, Clark N. Absconding: a literature review. J Psychiatr Ment Health Nurs 1998;5:343-53. 
3. Stewart D, Bowers L. Absconding from psychiatric hospitals: a literature review. 2010. http://www.kcl.ac.uk/ioppn/depts/hspr/ research/ciemh/mhn/projects/litreview/LitRevAbsc.pdf (accessed 12 Nov 2015).

4. Clark N, Kiyimba F, Bowers L, et al. Absconding: nurses views and reactions. J Psychiatr Ment Health Nurs 1999;6:219-24.

5. Martin T, Thomas S. Police officers' views of absconding from mental health units in Victoria, Australia. Int J Ment Health Nurs 2014;23:145-52.

6. Bowers L, Jarrett $\mathrm{M}$, Clark $\mathrm{N}$, et al. Absconding: outcome and risk. J Psychiatr Ment Health Nurs 1999;6:213-8.

7. Department of Health. Mental Health Act 1983: Code of Practice. 2015. Available at https://www.gov.uk/government/uploads/system/ uploads/attachment_data/file/435512/MHA_Code_of_Practice.PDF (accessed 17 May 2016)

8. Muir-Cochrane E, Mosel KA. Absconding: A review of the literature 1996-2008. Int J Ment Health Nurs 2008;17:370-8.

9. Meehan T, Morrison P, McDougall S. Absconding behaviour: an exploratory investigation in an acute inpatient unit. Aust N Z J Psychiatry 1999;33:533-7.
10. Bowers L, Jarrett M, Clark N, et al. Determinants of absconding by patients on acute psychiatric wards. J Adv Nurs 2000;32:644-9.

11. Macrae C. The problem with incident reporting. BMJ Qual Saf 2016;25:71-5

12. Vincent C. Incident reporting and patient safety. BMJ 2007;334:51.

13. Bowers L, Alexander J, Gaskell C. A trial of an anti-absconding intervention in acute psychiatric wards. J Psychiatr Ment Health Nurs 2003;10:410-6.

14. Bowers L, Simpson A, Alexander J. Real world application of an intervention to reduce absconding. J Psychiatr Ment Health Nurs 2005;12:598-602.

15. Mohammed MA, Worthington $P$. Why traditional statistical process control charts for attribute data should be viewed alongside an xmr-chart. BMJ Qual Saf 2013;22:263-9.

16. Brickell TA, Nicholls TL, Procyshyn RM, et al. Patient safety in mental health. 2009. http://www.patientsafetyinstitute.ca/en/ toolsResources/Research/commissionedResearch/ mentalHealthAndPatientSafety/Documents/Mental\%20Health\% 20Paper.pdf: Edmonton, Alberta: Canadian Patient Safety Institute and Ontario Hospital Association (accessed 15 Jan 2016). 